\title{
A SIMPLE PROOF OF CLARKSON'S INEQUALITY
}

\section{S. RAMASWAMY}

In [1], J. A. Clarkson has proved the following inequality.

Let $1<p \leqslant 2$. Let $f$ and $g$ be any two functions in $L^{p}[0,1]$. Then,

$$
2^{p-1}\left(\|f\|^{p}+\|g\|^{p}\right) \leqslant\|f+g\|^{p}+\|f-g\|^{p} \leqslant 2\left(\|f\|^{p}+\|g\|^{p}\right) .
$$

Actually, J. A. Clarkson first proves that

$$
\|f+g\|^{q}+\|f-g\|^{q} \leqslant 2\left(\|f\|^{p}+\|g\|^{p}\right)^{q-1}
$$

where $q$ is such that $1 / p+1 / q=1$. He then deduces inequality (1) from (2).

The proof of inequality (2) is rather long and nontrivial. Since, it is used to deduce (1), it appears that (1) is also complicated. Actually, the proof of (1) is quite easy and the purpose of this note is to give a simple proof of (1).

To prove (1), it is sufficient to prove only the right-hand side i.e., $\forall f, g \in$ $L^{p}[0,1],\|f+g\|^{p}+\|f-g\|^{p} \leqslant 2\left(\|f\|^{p}+\|g\|^{p}\right)$. Then, by applying this to the functions $h_{1}$ and $h_{2}$ where $h_{1}=f+g$ and $h_{2}=f-g$, we get the lefthand side of ( 1 ).

We shall establish the right-hand side of $(1)$, for $L^{p}(\Omega, \theta, \lambda)$ where $(\Omega, \theta, \lambda)$ is a measure space with $\lambda(\Omega)=1$.

Let $\varepsilon_{n}$ be the $n$th Rademacher function on $[0,1]$, i.e.

$$
\begin{aligned}
\varepsilon_{n}(t) & =(-1)^{k} \quad \text { if } t \in\left((k-1) / 2^{n}, k / 2^{n}\right) \text { for some } k \in\left\{1, \ldots, 2^{n}\right\} \\
& =0 \text { if } t=k / 2^{n} \text { for some } k \in\left\{0, \ldots, 2^{n}\right\} .
\end{aligned}
$$

Then it is clear that $\left|\varepsilon_{n}(t)\right|=1$ for almost all $t$ (with respect to the Lebesgue measure on $[0,1])$ and the measure of $\left\{t: \varepsilon_{n}(t)=+1\right\}=$ measure of $\{t$ : $\left.\varepsilon_{n}(t)=-1\right\}=\frac{1}{2}$.

It is clear that

$$
\begin{aligned}
\frac{1}{2}\left[\|f+g\|^{p}+\right. & \left.\|f-g\|^{p}\right]=\int_{0}^{1}\left\|\varepsilon_{1}(t) f+\varepsilon_{2}(t) g\right\|^{p} d t, \\
\int_{0}^{1}\left\|\varepsilon_{1}(t) f+\varepsilon_{2}(t) g\right\|^{p} d t & =\int_{0}^{1}\left(\int_{\Omega}\left|\varepsilon_{1}(t) f(w)+\varepsilon_{2}(t) g(w)\right|^{p} d \lambda(w)\right) d t \\
& =\int_{\Omega}\left(\int_{0}^{1}\left|\varepsilon_{1}(t) f(w)+\varepsilon_{2}(t) g(w)\right|^{p} d t\right) d \lambda(w) \\
& =\int_{\Omega}\left(\int_{0}^{1}\left\{\left|\varepsilon_{1}(t) f(w)+\varepsilon_{2}(t) g(w)\right|^{2}\right\}^{p / 2} d t\right) d \lambda(w) .
\end{aligned}
$$

Received by the editors December 8, 1976.

AMS (MOS) subject classifications (1970). Primary 46E30, 46G99; Secondary 46B99.

Key words and phrases. Rademacher function. 
Since $p / 2 \leqslant 1$, by Hölder's inequality, the right-hand side is

$$
\leqslant \int_{\Omega}\left(\int_{0}^{1}\left|\varepsilon_{1}(t) f(w)+\varepsilon_{2}(t) g(w)\right|^{2} d t\right)^{p / 2} d \lambda(w) .
$$

Using the fact that $\int\left|\varepsilon_{1}(t)\right|^{2} d t=\int\left|\varepsilon_{2}(t)\right|^{2} d t=1$ and $\int_{0}^{1} \varepsilon_{1}(t) \varepsilon_{2}(t) d t=0$,

$$
\int_{0}^{1}\left|\varepsilon_{1}(t) f(w)+\varepsilon_{2}(t) g(w)\right|^{2} d t=|f(w)|^{2}+|g(w)|^{2}
$$

Hence,

$$
\int_{0}^{1}\left\|\varepsilon_{1}(t) f+\varepsilon_{2}(t) g\right\|^{p} d t \leqslant \int_{\Omega}\left(|f(w)|^{2}+|g(w)|^{2}\right)^{p / 2} d \lambda(w) .
$$

Further, we have,

$$
\left(|f(w)|^{2}+|g(w)|^{2}\right)^{p / 2} \leqslant|f(w)|^{p}+|g(w)|^{p}, \quad \text { as }(1+t)^{r} \leqslant 1+t^{r}
$$

when $0<r \leqslant 1$ and $t \geqslant 0$. Hence, the right-hand side of (3) is

$$
\leqslant \int\left(|f(w)|^{p}+|g(w)|^{p}\right) d \lambda(w)=\|f\|^{p}+\|g\|^{p} .
$$

This establishes the Clarkson's inequality (1).

It is clear that (1) is true for functions belonging to $L^{p}$ of any finite measure space. Since the $L^{p}$ space of any $\sigma$-finite measure space is isometric to the $L^{p}$ space of a finite measure space, the inequality (1) obviously holds for functions belonging to the $L^{p}$ space of any $\sigma$-finite measure space.

Analogously, it can be proved that if $2 \leqslant p<\infty$ and if $f$ and $g$ are any two functions belonging to the $L^{p}$ space over any $\sigma$-finite measure space, then

$$
2\left(\|f\|^{p}+\|g\|^{p}\right) \leqslant\|f+g\|^{p}+\|f-g\|^{p} \leqslant 2^{p-1}\left(\|f\|^{p}+\|g\|^{p}\right) .
$$

That the inequality (1) of J. A. Clarkson can be derived as above was observed after going through [2], where it is proved that $L^{p}$ is type $p$-Rademacher for $1 \leqslant p \leqslant 2$.

ADDED IN PROOF. We remark that the above proof goes through as such even if $\lambda$ is only a $\sigma$-finite measure.

\section{REFERENCES}

1. J. A. Clarkson, Uniformly convex spaces, Trans. Amer. Math. Soc. 40 (1936), 396-414.

2. G. Pisier, "Type" des espaces normés, Seminaire Maurey-Schwartz 1973-1974; Exp. No. 3, Centre de Math., Ecole Polytech., Paris, 1974, 12 pp.

School of Mathematics, Tata Institute of Fundamental Research, Hom Bhabha Road, BOMBAY 400005, INDIA 